\title{
LINGUAGEM E APRENDIZAGEM: A ATENUAÇÃO EM PORTUGUÊS E ESPANHOL
}

\author{
LENGUAJE Y APRENDIZAJE: LA ATENUACIÓN EN PORTUGUÉS Y EN \\ ESPAÑOL ${ }^{1}$
}

\section{LANGUAGE AND LEARNING: MITIGATION IN PORTUGUES AND SPANISH}

\author{
Jorge MARTÍ CONTRERAS ${ }^{2}$
}

\begin{abstract}
RESUMO: Neste artigo, será desenvolvida uma análise interlinguística (espanholportuguês) para determinar os elementos de atenuação existentes em espanhol e português. Para isso, foi muito importante a importância dada ao fenômeno pragmático da atenuação no Quadro Europeu Comum de Referência para as línguas e no Plan Curricular do Instituto Cervantes. Depois se faz uma análise baseada em fundamentos teóricos sobre o que é a atenuação e quais são os recursos existentes na língua materna do aluno. A seguir, é colocado o Focus on Form e é facilitada a aquisição da L2 (também poderia ser aplicada ao contrário, para a aprendizagem do português de nativos espanhóis). Ao fim, é proposta uma sequência didática de uma análise contrastiva nos dois idiomas para a aprendizagem da atenuação.
\end{abstract}

PALAVRAS-CHAVE: Atenuação. Espanhol. Português. Língua estrangeira.

RESUMEN: En este artículo se va a desarrollar un análisis interlingüístico (españolportugués) e intralingüístico para determinar los elementos de atenuación que existen tanto en español como en portugués. Para esto, primero se ha tenido en cuenta cuál es la importancia que se le da al fenómeno pragmático de la atenuación en el Marco Común Europeo de Referencia y en el Plan Curricular del Instituto Cervantes, para después establecer un análisis que parte de una fundamentación teórica sobre qué es la atenuación y cuáles son los recursos que existen en la lengua materna del estudiante. Después, se pone el Foco en la Forma y se facilita la adquisición de la L2 (también se podría aplicar de forma inversa, para el aprendizaje de Portugués en nativos españoles). Finalmente, se propone una secuencia didáctica a través de un análisis contrastivo en las dos lenguas, para el aprendizaje de la atenuación

PALABRAS CLAVE: Atenuación. Español. Portugués. Lengua Extranjera.

\footnotetext{
${ }^{1}$ Este artículo ha sido posible gracias al apoyo de la OCIT (E-2018-09) de la Universitat Jaume I, y a la estancia de investigación con la profesora Isabel Margarida Oliveira Duarte en la Universidade do Porto. Este trabajo se enmarca en el proyecto La atenuación pragmática en su variación genérica: géneros discursivos escritos y orales en el español de España y América (FFI2016-75249-P), financiado por el Ministerio de Economía y Competitividad.

${ }^{2}$ Universitat Jaume I (UJI) - Castelló de la Plana - España. Profesor Ayudante Doctor, Dpto. Filología y Culturas Europeas. ORCID: https://orcid.org/0000-0002-1619-1839. E-mail: jmarti@uji.es
} 
ABSTRACT: In this paper, we will develop an interlinguistic (Spanish-Portuguese) and a intralinguistic analysis to stablish what elements could be mitigators in Spanish and in Portuguese. First it has been taken into account what is the importance given to the pragmatic phenomenon of mitigation in the Common European Framework of Reference and in the Plan Curricular (Instituto Cervantes). Then, we establish an analysis based on the literature about the mitigation and what resources exist in the student's mother tongue. After that, we Focus on Form and in the learning process (it could be also applied in reverse, for learning Portuguese in Spanish natives). Finally, we propose a didactic sequence with a contrastive analysis in both languages, for learning mitigation.

KEYWORDS: Mitigation. Spanish. Portuguese. Second Language

\section{Introducción}

Tanto las instituciones como los centros educativos suelen tener problemas a la hora de realizar los programas formativos (sobre todo por el límite temporal de los cursos), ya que, o bien se centran en objetivos puramente gramaticales, o bien en contenidos funcionales o comunicativos generales, y no tienen en cuenta aspectos lingüísticos y pragmáticos que pueden ayudar a alcanzar el objetivo comunicativo que es el que establece el Marco Común Europeo de Referencia (CONSEJO DE EUROPA, 2002); y tampoco se suele estudiar de forma directa el uso oral real de los hablantes nativos, ya que se suele emplear material audiovisual diseñado por los autores de los materiales didácticos. Este material no presenta un lenguaje oral y espontáneo de una conversación coloquial (o semiformal o formal), sino que se fuerzan las expresiones empleadas en el discurso oral para que aborden los objetivos gramaticales que se deben dar en la unidad didáctica.

Existen corpus lingüísticos de conversaciones orales reales y espontáneas. En portugués se pueden destacar: Projeto Fala Bracarense de la Universidade do Minho, el corpus Banco de Português del proyecto DIRECT (LAEL, PUCSP) y Linguateca. En el ámbito del español, son consultas obligatorias el elaborado por el grupo de investigación Valesco (encabezado por el Dr. Antonio Briz; recoge conversaciones coloquiales de Valencia), el corpus Es.Vag.Atenuación (dirigido por la Dra. Marta Albelda y por la Dra. María Estellés, está compuesto por conversaciones coloquiales de España y de América Latina) y el CREA (Corpus de Referencia del Español Actual, elaborado por la Real Academia de la Lengua). Gracias a estos corpus se puede llevar al aula material oral real y se pueden trabajar aspectos pragmáticos como la atenuación. En 
definitiva, muy pocos manuales abordan contenidos de atenuación o de intensificación de forma directa.

\section{La atenuación en el Marco Común Europeo y en el Plan Curricular}

Las dos obras básicas que sirven como fundamentación teórica a la hora de que el profesorado deba elaborar las secuencias didácticas que llevará al aula son dos, El Marco Común Europeo de Referencia (CONSEJO DE EUROPA, 2002) en donde la atenuación lingüística no viene reflejada directamente. $\mathrm{Y}$ el Plan Curricular (INSTITUTO CERVANTES, 2007) en el que sí que aparece un capítulo dedicado a las "tácticas y estrategias pragmáticas" (cap. 6). Y dentro de este capítulo, un breve apartado que solo trata la cortesía verbal atenuadora (y no tiene en cuenta otros tipos de atenuación) que a su vez subdivide en: atenuación del papel del hablante o del oyente, atenuación del acto amenazador y atenuación dialógica. Presentamos en la siguiente tabla los rasgos y recursos descritos para cada nivel:

Tabla 1 - Ejemplos del Plan Curricular

\begin{tabular}{|c|c|c|}
\hline Rasgo & Nivel & Descripción de los recursos empleados \\
\hline \multirow{6}{*}{$\begin{array}{l}\text { Atenuación del } \\
\text { papel del } \\
\text { hablante o del } \\
\text { oyente. } \\
\text { Desplazamiento } \\
\text { pronominal de la } \\
\text { 2. persona }\end{array}$} & A1 & A $3 .^{a}$ persona: uso de usted \\
\hline & A2 & A estructuras con valor impersonal. ¿Se puede?, Hay que estudiar \\
\hline & B1 & A 1. a persona de singular. Yo en tu lugar..., Yo que tú \\
\hline & B2 & 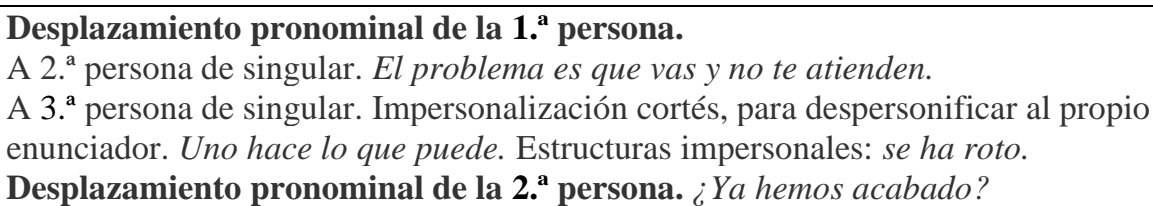 \\
\hline & $\mathrm{C} 1$ & $\begin{array}{l}\text { Desplazamiento pronominal de la } \mathbf{1}^{\text {a }} \text { persona. } \\
\text { A 1. }{ }^{\text {a }} \text { persona de plural. Nos hemos equivocado. } \\
\text { A 2. }{ }^{\text {a }} \text { persona. Para mitigar el acto: mañana aquí me tenéis. } \\
\text { A 3. }{ }^{\text {a }} \text { persona. Impersonalización cortés: El que suscribe. } \\
\text { Desplazamiento pronominal de la } 2 .^{\text {a }} \text { persona. } \\
\text { A 1. }{ }^{\text {a }} \text { persona de plural: iA ver si nos callamos! } \\
\text { A estructuras pasivas sin explicitar el agente }\end{array}$ \\
\hline & $\mathrm{C} 2$ & 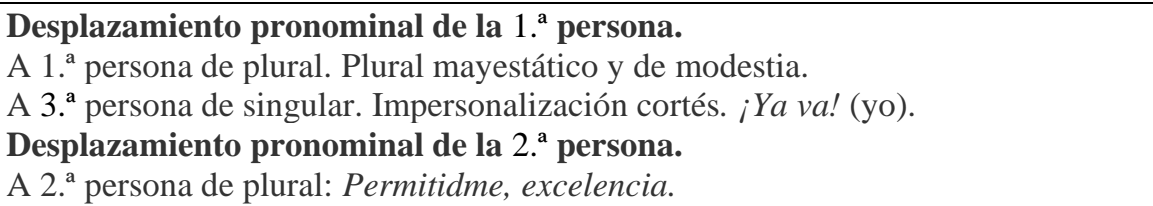 \\
\hline $\begin{array}{l}\text { Atenuación del } \\
\text { acto amenazador }\end{array}$ & A1 & $\begin{array}{l}\text { Verbo creer (forma afirmativa) para atenuar valoraciones o información } \\
\text { Formas rituales: Por favor }\end{array}$ \\
\hline
\end{tabular}




\begin{tabular}{|c|c|c|}
\hline & $\mathrm{A} 2$ & Actos de habla indirectos: ¿Me das el pan? \\
\hline & B1 & $\begin{array}{l}\text { Tácticas de desplazamiento de la perspectiva temporal. ¿Qué quería? } \\
\text { Condicional de cortesía. ¿Podrías cerrar la ventana? } \\
\text { Condicional de modestia. Yo diría que eso no es así } \\
\text { Perífrasis de futuro para rechazar. No sé si voy a poder ir. } \\
\text { Actos de habla indirectos } \\
\text { Verbos performativos para atenuar opiniones, creencias, afirmaciones } \\
\text { Reparaciones en las excusas (Es que...) y en las justificaciones (no fui porque...) } \\
\text { Infinitivo den con valor de mandato generalizador } \\
\text { No fumar }\end{array}$ \\
\hline & B2 & $\begin{array}{l}\text { Tácticas de desplazamiento de la perspectiva temporal. Condicional de cortesía, para } \\
\text { aconsejar o criticar, con verbos como querer, poder, valer, deber, convenir, necesitar, } \\
\text { desear. } \\
\text { Imperfecto de cortesía, para aconsejar o criticar } \\
\text { Futuro simple, para retrasar la respuesta o la acción ante una petición } \\
\text { Imperfecto de subjuntivo de cortesía } \\
\text { Verbos performativos para atenuar mandatos (desear, rogar, agradecer.) } \\
\text { Enunciados preliminares para anunciar un acto de habla que atenta contra la imagen } \\
\text { negativa del destinatario } \\
\text { ¿Puedo hacerte una pregunta indiscreta? } \\
\text { Formas rituales: Si no es molestia. }\end{array}$ \\
\hline & $\mathrm{C} 1$ & $\begin{array}{l}\text { Tácticas de desplazamiento temporal. Futuro de cortesía, futuro de modestia y actos de } \\
\text { habla indirectos } \\
\text { Interrogaciones introducidas por verbos volitivos o de posibilidad: querer, poder. } \\
\text { Formas rituales: ¿Me puede hacer el favor de...? } \\
\text { Eufemismos } \\
\text { Enunciados suspensivos y elipsis }\end{array}$ \\
\hline & $\mathrm{C} 2$ & $\begin{array}{l}\text { Tácticas de desplazamiento temporal. Pluscuamperfecto de cortesía: Me habías } \\
\text { preguntado algo, ¿verdad? } \\
\text { Cameladores. Tú que sabes tanto... }\end{array}$ \\
\hline $\begin{array}{l}\text { Atenuación } \\
\text { dialógica }\end{array}$ & B1 & $\begin{array}{l}\text { Para expresar acuerdo parcial, como preludio a un movimiento contraargumentativo } \\
\text { Tienes razón, pero a mí eso ya no me convence }\end{array}$ \\
\hline & B2 & $\begin{array}{l}\text { Para expresar incertidumbre, ignorancia o incompetencia ante lo dicho por el } \\
\text { interlocutor. Es posible que esté usted equivocado... } \\
\text { Para impersonalizar el desacuerdo. Es que... uno no va diciendo... }\end{array}$ \\
\hline & $\mathrm{C} 1$ & $\begin{array}{l}\text { Para expresar acuerdo parcial, como preludio a un movimiento contraargumentativo: } \\
\text { No, no es eso exactamente. } \\
\text { Para expresar incertidumbre, ignorancia o incompetencia. Me parece que te } \\
\text { equivocas... } \\
\text { Para minimizar el desacuerdo. Bueno, no creas. } \\
\text { Para introducir un acto de habla previo, en solicitud de disculpa: Sin ánimo de } \\
\text { contradecir }\end{array}$ \\
\hline & $\mathrm{C} 2$ & Para repetir una idea negada: Hombre, llover, llover, no llovió. \\
\hline
\end{tabular}

\section{Aproximación teórica al concepto de atenuación en la conversación}

La atenuación, según Briz (2005, p. 56), como estrategia pragmalingüística es una operación lingüística estratégica de minimización de lo dicho, vinculada a la 
actividad argumentativa y de negociación del acuerdo, que es el fin último de toda conversación. Mitigar, suavizar, disminuir la fuerza ilocutiva, reparar, esconder la verdadera intención; son los objetivos prácticos de la atenuación.

La atenuación es básicamente oral, de ahí la necesidad de que sea un recurso lingüístico espontáneo, ya que la conversación (Cestero Mancera, 2016, p. 2):

es la forma más natural, habitual y espontánea de comunicación interactiva humana, y la constatación empírica de que únicamente a través de la interacción comunicativa oral y, más concretamente, de la práctica conversacional es posible adquirir gran parte de las estructuras sintácticas complejas de una lengua, la mayoría de sus elementos discursivos o conversacionales y las funciones y actos comunicativos lingüístico-pragmáticos.

\section{Enseñanza de la atenuación Español como Lengua Extranjera}

En la enseñanza de E/LE surgen diferentes preguntas a las que vamos a dar solución:

- ¿Cuáles son los recursos de atenuación más empleados en español?

- ¿Qué estrategias podemos emplear en la enseñanza de la atenuación en los niveles B1 y B2? (como se ha visto en la tabla precedente de recursos atenuadores existen muchos recursos verbales, y vamos a ampliar la enumeración de elementos atenuadores a campos fuera de la conjugación verbal)

- ¿Existe similitud entre la atenuación en español y portugués?

Hemos clasificado en la siguiente tabla los rasgos atenuadores que se deben adquirir en los niveles B1 y B2 de Español y hemos buscado de los corpora lingüísticos citados en la primera parte del artículo, ejemplos que ilustren y aclaren cada uno de los rasgos. Por último, hemos añadido ejemplos también en portugués, ya que las actividades finales están dirigidas al estudiantado portugués. 
Tabla 2 - Rasgos atenuadores en B1 y B2

\begin{tabular}{|c|c|c|}
\hline Recurso $^{3}$ & Portugués & Español \\
\hline $\begin{array}{l}\text { 1. Modificadores morfológicos } \\
\text { internos: sufijos diminutivos }\end{array}$ & $\begin{array}{l}\text { (LAS: 143) «então fale um } \\
\text { pouquinho sobre futebol...» } \\
\text { (YO: } 227 \text { ) «Que ótimo! Fica bem } \\
\text { pertinho da minha casa!» } \\
\text { (CGFS) «a verdade é que não } \\
\text { tenho planos porque estou um } \\
\text { bocadinho confusa com a minha } \\
\text { vida...» }\end{array}$ & $\begin{array}{l}\text { [bien puestecito bien } \\
\text { presentadito] }\end{array}$ \\
\hline $\begin{array}{l}\text { 2. Modificadores externos: } \\
\text { cuantificadores } \\
\text { minimizadores, aproximativos } \\
\text { o difusores significativo } \\
\text { proposicionales } \\
\text { extraproposicionales }\end{array}$ & $\begin{array}{l}\text { (YO: } 227 \text { ) «Que ótimo! Fica bem } \\
\text { pertinho da minha casa!» } \\
\text { (LAS: 146) «olha... salão de } \\
\text { beleza para homem... isso é um } \\
\text { negócio meio afrescalhado... eu } \\
\text { eu eu não não iría no salão de } \\
\text { beleza pra homens agora cortar o } \\
\text { cabelo...» } \\
\text { (RC: 240) «Oi, sabe aquele } \\
\text { relatório mensal que você fez... } \\
\text { vai ter que refazer pois tem } \\
\text { algumas coisas que não estão } \\
\text { boas» }\end{array}$ & $\begin{array}{l}\text { eh involucrarloh a todoh un } \\
\text { poco }\end{array}$ \\
\hline 3. Lítotes y eufemismos & $\begin{array}{l}\text { (LAS: 146) «olha... salão de } \\
\text { beleza para homem... isso é um } \\
\text { negócio meio afrescalhado... eu } \\
\text { eu eu não não iría no salão de } \\
\text { beleza pra homens agora cortar o } \\
\text { cabelo...» } \\
\text { (RC: 239) «precisariam ser } \\
\text { reescritos...» } \\
\text { (RC: 239) } \text { modificar o relatório» } \\
\text { (RC: 240) «Oi, sabe aquele } \\
\text { relatório mensal que você fez... } \\
\text { vai ter que refazer pois tem } \\
\text { algumas coisas que não estão } \\
\text { boas» }\end{array}$ & $\begin{array}{l}\text { [porque igual] no es tan fa- } \\
\text { bueno }\end{array}$ \\
\hline $\begin{array}{l}\text { 4. Empleo de palabras } \\
\text { extranjeras }\end{array}$ & & Lo dijo el man. \\
\hline $\begin{array}{l}\text { 5. Usos modalizados de los } \\
\text { tiempos verbales }\end{array}$ & $\begin{array}{l}\text { (CGFS) «E } 8 \text {-) queria acrescentar } \\
\text { também qu faz que as pessoas } \\
(\ldots) \text { } \\
\text { (LAS: } 143 \text { ) «porque se não } \\
\text { tivesse o servente que varresse a } \\
\text { rua não tivesse o servente que }\end{array}$ & $\begin{array}{l}\text { [¿y a qué- a qué grupo] se le } \\
\text { daría eso a todoh? }\end{array}$ \\
\hline
\end{tabular}

${ }^{3}$ Los ejemplos en portugués recogidos en esta tabla proceden de los trabajos de Da Silva (2017), y los hemos señado como (LAS), de Gomes y Silva, que aparecen como (CGFS), de Topa (HT), Oliveirea (YO) y Caggiano (RC) los ejemplos en español pertenecen al proyecto ES.VAR.ATENUACION La enumeración de recursos los hemos tomado de Briz y Albelda (2013) y de Albelda et al. (2014) 


\begin{tabular}{|c|c|c|}
\hline & $\begin{array}{l}\text { varresse a } \\
\text { repartição não seria:: quem é } \\
\text { que iria varrer a repartição? } \\
\text { seria o chefe da seção?» }\end{array}$ & \\
\hline $\begin{array}{l}\text { 6. Verbos, construcciones } \\
\text { verbales y partículas } \\
\text { discursivas modales que } \\
\text { expresan opinión en forma de } \\
\text { duda o de probabilidad }\end{array}$ & $\begin{array}{l}\text { (CGFS) «a minha situação é igual } \\
\text { que a sua mas possivelmente } \\
\text { vou ficar em casa...» } \\
\text { (HT: 130) «JS: (sobre definição } \\
\text { de "situação de carência") Um } \\
\text { casal de } 80 \text { anos que vivam os } \\
\text { dois } \\
\text { sozinhos com pensão mínima é } \\
\text { uma situação de carência? } \\
\text { AC: Com certeza. Nesses casos } \\
\text { posso-lhe garantir que não } \\
\text { haverá provavelmente } \\
\text { atualização } \\
\text { nenhuma [da renda].» }\end{array}$ & $\begin{array}{l}\text { me imagino que que cerraran } \\
\text { tarde Éxito ese día ¿no? }\end{array}$ \\
\hline $\begin{array}{lrr}7 . \quad \text { Verbos, } & \text { construcciones } \\
\text { verbales y y partículas } \\
\text { discursivas } \text { modales que } \\
\text { expresan fingimiento de } \\
\text { incertidumbre, } \\
\text { incompetencia o de ignorancia }\end{array}$ & & $\begin{array}{l}\text { que ((hahta)) me daba vergüenza } \\
\text { lo que había ehcrito y qué sé yo }\end{array}$ \\
\hline $\begin{array}{l}\text { 8. Construcciones acotadoras } \\
\text { de opinión a la propia persona } \\
\text { o a un determinado ámbito }\end{array}$ & $\begin{array}{l}\text { (CGFS) «no entanto eu acho que } \\
\text { há outras ...» } \\
\text { (CGFS) «Boa tarde, na minha } \\
\text { opinião acho que...» } \\
\text { (LAS: } 141 \text { ) «tu achas que alguma } \\
\text { vez o rádio assim pode: se } \\
\text { constituir um fator de atrito e de } \\
\text { briga?» }\end{array}$ & $\begin{array}{l}\text { es muy- para mí Chile es como } \\
\text { algo intermedio } \\
\text { y de hecho es que creo que es la } \\
\text { primera vez que te digo que } \\
\text { cambies de fecha }\end{array}$ \\
\hline $\begin{array}{l}\text { 9. Peticiones, preguntas, } \\
\text { mandatos úrdenes } \\
\text { expresados de forma indirecta }\end{array}$ & $\begin{array}{l}\text { (YO: 224) «A: Alô, Marisa está? } \\
{[\ldots]} \\
\text { B: um momento, por favor. Vou } \\
\text { chamá-la. » } \\
\text { (YO: 224): «Ganhei duas } \\
\text { entradas para o teatro. Não quer } \\
\text { ir comigo?» }\end{array}$ & $\begin{array}{l}\text { ¡ayyy>! lo siento cariño míoo } \\
\text { es un cinturón asesino }\end{array}$ \\
\hline 10. Expresiones de disculpa & & $\begin{array}{l}\text { No suma. Lo siento, pero no } \\
\text { suma. }\end{array}$ \\
\hline $\begin{array}{l}\text { 11. Estructuras sintácticas que } \\
\text { restringen el acto de habla }\end{array}$ & $\begin{array}{l}\text { LAS: 142) «sem esse primeiro } \\
\text { estudo de mensuração não se } \\
\text { sabe dizer se tem ou não tem } \\
\text { advogados demais ou se tem } \\
\text { médicos ou:: ou:: } \\
\text { dentistas de menos... é meio } \\
\text { difícil de dizer...» }\end{array}$ & $\begin{array}{l}\text { [gran diferencia] entonces } \\
\text { entiendo que hasta entre } \\
\text { españoles entonces ellos noo }\end{array}$ \\
\hline
\end{tabular}




\begin{tabular}{|c|c|c|}
\hline $\begin{array}{l}12 . \quad \text { Construcciones } \\
\text { justificadoras o de excusa }\end{array}$ & & $\begin{array}{l}\text { lo que pasa es que no te dan cita } \\
\text { lo que tú tienes que hacer es ir y } \\
\text { pararte y hacer la cola }\end{array}$ \\
\hline $\begin{array}{l}\text { 13. Elipsis de la conclusión, } \\
\text { estructuras suspendidas o } \\
\text { estructuras truncadas }\end{array}$ & & $\begin{array}{l}\text { hemos de tranquilizar a todo el } \\
\text { mundo: a los padres, a las } \\
\text { personas que aún buscan } \\
\text { empleo, a los empresarios y a los } \\
\text { inversores, a los pensionistas, a } \\
\text { la Unión Europea... a todo el } \\
\text { mundo. }\end{array}$ \\
\hline 14. Impersonalización & & $\begin{array}{l}\text { lo que entonces uno se pregunta } \\
\text { ¿por qué se entienden si utilizan } \\
\text { mucho menos pero iguall [se } \\
\text { puedee-?] }\end{array}$ \\
\hline $\begin{array}{l}\text { 15. Impersonalización a través } \\
\text { del estilo directo }\end{array}$ & $\begin{array}{l}\text { (CGFS) «é sabido que muita } \\
\text { gente diz que esta disciplina não } \\
\text { é muito importante, mas } \\
\text { ehhh...» }\end{array}$ & $\begin{array}{l}\text { Todo el mundo sabe que no } \\
\text { cabe gobernar con varios } \\
\text { criterios a la vez }\end{array}$ \\
\hline $\begin{array}{l}\text { 16. Movimiento de } \\
\text { reformulación (con partícula, } \\
\text { sin partícula o con cambio de } \\
\text { tópico) }\end{array}$ & & $\begin{array}{l}\text { pero bueno eso tiene que tener } \\
\text { un tiempo no le va' a dar- no sé }\end{array}$ \\
\hline 17. Concesividad & $\begin{array}{l}\text { (LAS: 146) «ecentemente eu eu } \\
\text { acho eu acho muito bom tem } \\
\text { algumas coisas que são } \\
\text { inovações boas mas esse } \\
\text { negócio de salão de beleza pra } \\
\text { homem eh:: é meio } \\
\text { afrescalhado...» }\end{array}$ & no sé qué se habla pero digo \\
\hline $\begin{array}{l}\text { 18. Partículas discursivas } \\
\text { modales de objetivación }\end{array}$ & & $\begin{array}{l}\text { [qué era] alguna estupideh- } \\
\text { porque la verdad que a mí no } \\
\text { me parece nada [divertido] }\end{array}$ \\
\hline $\begin{array}{l}\text { 19. Partículas discursivas y } \\
\text { expresiones de control de } \\
\text { contacto con el interlocutor }\end{array}$ & $\begin{array}{l}\text { (LAS: 145) «e aí a gente mantém } \\
\text { uma conversa durante quarenta } \\
\text { minutos... não é?» } \\
\text { (LAS: 145) «você podia por } \\
\text { exemplo descrever isso aqui... } \\
\text { esse lugar onde você mora... } \\
\text { né?... e situar isso dentro da } \\
\text { cidade do Rio de Janeiro... tá?» }\end{array}$ & $\begin{array}{l}\text { cogieron la guagua a tiempo } \\
\text { ¿no? }\end{array}$ \\
\hline $\begin{array}{l}\text { 20. Formas de tratamiento y } \\
\text { fórmulas apelativa. }\end{array}$ & $\begin{array}{l}\text { (LAS: } 145) \text { «Como é que } \\
\text { está... eh... estruturado o ensino, } \\
\text { quais os níveis de ensino que nós } \\
\text { temos aqui... eh... entre nós?» } \\
(\mathrm{RC}: \quad 244) \quad \text { Gente, vamos } \\
\text { tentar»/ «olha, acho que...»/ } \\
\text { «Fulano, encontrei...»/ «Oi, } \\
\text { sabe aquele relatório...»/ }\end{array}$ & $\begin{array}{l}\text { Ustedes y yo sabemos que no es } \\
\text { razonable }\end{array}$ \\
\hline
\end{tabular}


A la hora de analizar las conversaciones coloquiales y en el momento de llevar al aula las secuencias didácticas se deben establecen unos patrones comunes que ayuden a analizar los contextos situacionales y a dilucidar si los recursos empleados son atenuadores o no, por ejemplo, el sufijo diminutivo en (1), en el caso a) es atenuador y en el b) no lo es.

(1) a. Le he dado un golpecito al coche.

b. He comprado un lapicito, mira qué pequeño es.

También se debe tener en cuenta que existen tres partes que intervienen en la atenuación (BRIZ; ALBELDA, 2013, p. 301): el segmento desencadenante (lo que favorece o provoca la atenuación), el elemento o segmento atenuante (lo que atenúa) y el segmento atenuado, lo que se atenúa. Si no aparecen en el contexto estos tres elementos es posible que no estemos delante de una atenuación, sino de otro recurso pragmático.

\section{Propuesta didáctica}

Profesorado de Portugués como Lengua Extranjera (PLE) de la Universidade do Porto (GOMES; SILVA, 2014, p. 56) ha publicado material didáctico para la enseñanza de PLE para trabajar tanto la atenuación como la intensificación. Nosotros tomamos la estructura básica del diseño de la secuencia didáctica para formular las actividades en español.

1) Localiza los elementos atenuadores en portugués en el siguiente texto ${ }^{4}$, después tradúcelos al español.

1. B: 个ela a mim dizia-me uma bez eu bi lá umas botas que gostei muito e ela disse entra Fatinha// ai eu posso entrar mas nem penses [que]

2. A: [(RISOS)]

3. B: diz ela// digo assim $\uparrow$ quanto é que custam aquelas botas? são giras/ não são? $\downarrow$ digo assim são/ duzentos e não sei- oh oh// Carminho/ por amor de Deus// eu não sou rica

4. A: (RISOS)

5. B: diz ela assim/ ah mas tu podes pagar em duas ou três bezes/ não Carminho/ não/ nem pensar/ não quero $\downarrow$ [obrigada]/ [mesmo] pagando em duas e três [vezes] [não] Carminho/ não/ nem pensar/ não quero $\downarrow$ [obrigada]

6. A: [mesmo] pagando em duas e três [vezes]

7. B: [não]

8. A: =não deixa de ser [um total]

9. B: [não não]

10. A: =não deixa de ser duzentos

11. B: não não/ Carminho

${ }^{4}$ Texto extraído de Duarte (2015, p. 65) 
12. A: (RISOS)

13. B: isso é p(ara)os teus clientes/ tu é que tens aqui clientes muito ricos/ eu não sou rica [e ela]

14. A: $[\operatorname{sim}] /$ mas pronto era- era- era- era o- era o negócio dela

$15 \mathrm{~B}: \operatorname{sim} / \mathrm{sim}$.

2) Clasifica los elementos atenuadores del texto anterior en la siguiente tabla:

Tabla 3 - Ejemplo de actividad

\begin{tabular}{|c|c|c|}
\hline \multirow{2}{*}{\multicolumn{3}{|c|}{$\begin{array}{l}\text { Recurso } \\
\text { 1. Diminutivos }\end{array}$}} \\
\hline & & \\
\hline \multicolumn{3}{|l|}{$\begin{array}{l}\text { 2. Cuantificadores minimizadores, } \\
\text { aproximativos o difusores }\end{array}$} \\
\hline \multicolumn{3}{|l|}{ 3. Lítotes y eufemismos } \\
\hline \multicolumn{3}{|l|}{ 4. Empleo de palabras extranjeras } \\
\hline \multicolumn{3}{|l|}{$\begin{array}{l}\text { 5. Usos modalizados de los tiempos } \\
\text { verbales }\end{array}$} \\
\hline \multicolumn{3}{|l|}{$\begin{array}{l}\text { 6. Verbos o construcciones verbales que } \\
\text { expresan opinión en forma de duda o de } \\
\text { probabilidad }\end{array}$} \\
\hline \multicolumn{3}{|l|}{$\begin{array}{l}\text { 7. Verbos o construcciones que expresan } \\
\text { fingimiento de incertidumbre, de } \\
\text { incompetencia o de ignorancia }\end{array}$} \\
\hline \multicolumn{3}{|l|}{$\begin{array}{l}\text { 8. Construcciones acotadoras de opinión } \\
\text { a la propia persona o a un determinado } \\
\text { ámbito }\end{array}$} \\
\hline \multicolumn{3}{|l|}{$\begin{array}{l}\text { 9. Peticiones, preguntas, mandatos } \mathrm{u} \\
\text { órdenes expresados de forma indirecta }\end{array}$} \\
\hline \multicolumn{3}{|l|}{ 10. Expresiones de disculpa } \\
\hline \multicolumn{3}{|l|}{$\begin{array}{l}\text { 11. Estructuras sintácticas que restringen } \\
\text { el acto de hablan }\end{array}$} \\
\hline \multicolumn{3}{|l|}{$\begin{array}{l}\text { 12. Construcciones justificadoras o de } \\
\text { excusa }\end{array}$} \\
\hline \multicolumn{3}{|l|}{$\begin{array}{l}\text { 13. Elipsis de la conclusión, estructuras } \\
\text { suspendidas o estructuras truncadas }\end{array}$} \\
\hline \multicolumn{3}{|l|}{ 14. Impersonalización } \\
\hline \multicolumn{3}{|l|}{$\begin{array}{l}\text { 15. Impersonalización a través del estilo } \\
\text { directo }\end{array}$} \\
\hline \multicolumn{3}{|l|}{ 16. Movimiento de reformulación } \\
\hline \multicolumn{3}{|l|}{ 17. Concesividad } \\
\hline \multicolumn{3}{|l|}{$\begin{array}{l}\text { 18. Partículas discursivas modales de } \\
\text { objetivación }\end{array}$} \\
\hline \multicolumn{3}{|l|}{$\begin{array}{l}\text { 19. Partículas discursivas y expresiones } \\
\text { de control de contacto con el interlocutor }\end{array}$} \\
\hline $\begin{array}{l}\text { 20. Formas de tratamiento y fórmulas } \\
\text { apelativas. }\end{array}$ & & \\
\hline
\end{tabular}


3) En parejas, escribid un diálogo en español en el que se empleen las mismas estructuras, pero inventad ejemplos nuevos.

4) Vea el siguiente fragmento de la televisión portuguesa RTP sobre las elecciones presidenciales de Portugal de 2011 (http://www.rtp.pt/programa/tv/p27111) y añada en la tabla del ejercicio 3 cuatro nuevos ejemplos de atenuación. Después, mire el debate sobre las elecciones presidenciales de España de 2019 (http://www.rtve.es/noticias/elecciones/generales/debates/) y añada cuatro nuevos ejemplos.

5) Tarea de producción oral. El alumnado de la clase se divide en cuatro grupos. Dos de ellos se enfrentarán en un debate y los otros dos grupos analizarán el discurso empleado por los compañeros y comprobarán si han empleado, o no, los recursos atenuadores necesarios. Antes del debate, los grupos tendrán tiempo suficiente para preparar las intervenciones. Cuando acaba el primer debate, el grupo observador pasará a ser el protagonista del debate, y el otro grupo asumirá el papel de evaluador.

6) Tarea de producción escrita. Escribe un diálogo inventado en español sobre una discusión que ha tenido lugar durante una entrevista radiofónica y en donde el entrevistador está encontrando incongruencias en la argumentación del invitado y el entrevistado explica sus motivos para esgrimir estas mentiras. Texto de aproximadamente 200 palabras.

\section{Consideraciones finales}

A lo largo del artículo se han analizado las obras básicas relacionadas con la enseñanza de lenguas, el nivel lingüístico que el alumnado debe adquirir en cada momento de su formación y los recursos lingüísticos y pragmáticos que se han de asimilar durante el aprendizaje de una lengua.

Aunque el Marco Común Europeo de Referencia no hace ninguna alusión al aprendizaje de un elemento pragmático tan habitual en la conversación oral espontánea, hemos visto que es un recurso muy utilizado tanto en español como en portugués y ambas lenguas tienen los mismos fenómenos pragmáticos y recursos lingüísticos; esto 
facilita mucho la enseñanza de una segunda lengua, por lo tanto, abogamos por la enseñanza de recursos estilísticos a través de la comparación de lenguas.

AGRADECIMENTOS: Este artículo ha sido posible gracias al apoyo de la OCIT (E2018-09) de la Universitat Jaume I, y a la estancia de investigación con la profesora Isabel Margarida Oliveira Duarte en la Universidade do Porto. Este trabajo se enmarca en el proyecto La atenuación pragmática en su variación genérica: géneros discursivos escritos y orales en el español de España y América (FFI2016-75249-P), financiado por el Ministerio de Economía y Competitividad.

\section{REFERENCIAS}

ALBELDA MARCO, Marta. et al. Ficha metodológica para el análisis pragmático de la atenuación en corpus discursivos orales. Oralia, núm. 17, p. 7-62, 2014.

ALBELDA MARCO, Marta; ESTELLÉS ARGUEDAS, María et al. Corpus propio de conversaciones coloquiales en español de España y de América, 2019.

http://esvaratenuacion.es/corpus-discursivo-propio/

\section{BRIZ, Antonio. El español coloquial en la conversación: Esbozo de pragmagramática. Barcelona: Ariel, 1998.}

BRIZ, Antonio; ALBELDA, Marta. Una propuesta teórica y metodológica para el análisis de la atenuación lingüística en español y portugués. La base de un proyecto en común ES.VAR.ATENUACIÓN [en línea]. Onomázein, 28, p. 288-319, 2013. http://onomazein.letras.uc.cl/Articulos/N28/28-23_Briz_FINAL.pdf

CAGGIANO BLANCO, Ramiro Carlos Humberto. Atenuação pragmática e problemas de intercompreensão: um estudo intercultural entre universitários paulistanos e cordobeses. Revista ELUA, Anexo IV, p. 231-249, 2018.

CESTERO MANCERA, Ana María. La conversación. Bases teóricas y metodológicas para su investigación y su enseñanza en ELE. Linred, núm. 5, p. 1-17, 2016.

http://www.linred.es/articulos_pdf/LR_articulo_24042007.pdf

CONSEJO DE EUROPA. Marco Común Europeo de Referencia para las Lenguas: aprendizaje, enseñanza, evaluación. Madrid: MECD y Grupo Anaya, 2002 [2001]. https://cvc.cervantes.es/ensenanza/biblioteca_ele/marco/cvc_mer.pdf

DA SILVA, Luiz Antônio. Atenuação em entrevistas do projeto NURC. Normas, n. 7, p. 135-148, 2017. http://dx.doi.org/10.7203/Normas.7.10429

DE OLIVEIRA CAGGIANO BLANCO, Yedda. Alves. A relação entre imagem e atenuação na análise de materiais de ple. Revista ELUA, Anexo IV, p. 215-229, 2018. 
DUARTE, Isabel. Textos orais: análise da conversa informal e ensino do Português Língua Estrangeira. Todas as letras, v. 17, n. 1, p. 56-723, 2015.

GOMES, Carlos; SILVA, Fátima. Mecanismos de atenuação e intensificação no ensino-aprendizagem do português como língua estrangeira. Redis: revista de estudos do discurso, n. 3, p. 32-66, 2014.

INSTITUTO CERVANTES. Plan curricular. Madrid: Biblioteca Nueva, 2007.

TOPA, Helena. Quando a reformulação é uma remodalização com efeito de atenuação. Normas, n. 7, p. 126-134, 2017. http://dx.doi.org/10.7203/Normas.7.10428

\section{Cómo citar este artículo}

MARTÍ-CONTRERAS, Jorge. Linguagem e aprendizagem: a atenuação em Portuguës e Espanyol. Rev. EntreLínguas, Araraquara, v. 5, n. esp. 1, p. 344-356, jul., 2019. EISSN: 2447-3529. DOI: 10.29051/el.v5iesp.1.12989

Submetido em: 01/06/2019

Aprovado em: 30/06/2019

Publicado em: 01/10/2019 